\title{
La nueva lingüística del habla
}

\section{The new linguistics of speech}

\author{
Heinrich Helberg Chávez \\ Universidad Nacional Mayor de San Marcos \\ hhelberg@hotmail.com
}

\section{Resumen}

El presente artículo pretende formular los fundamentos de una nueva lingüística que se centra en la experiencia cotidiana del habla y niega la existencia de códigos como medio de explicación de la comprensión del lenguaje y les da solo un valor simbólico. En su lugar, la nueva lingüística desarrolla una hermenéutica interactiva para comprender los mecanismos del habla, la adquisición del lenguaje y su lógica práctica que es constitutiva para el universo de sentido, puesto que hablamos como aprendimos a hacerlo. La nueva lingüística se centra en los intereses del hablante que quiere ser dueño de su manera de hablar y definir los sentidos en situaciones concretas, en contraste con una lingüística que defiende los intereses nacionales simbolizados en los códigos compartidos.

Palabras clave: Lingüistica teórica, filosofía del lenguaje, lenguaje, habla

\section{Abstract}

The present article aims to formulate the fundamentals of a new linguistics that is focused on the daily experience of speech and denies the existence of codes as an explaining devise of language understanding and gives them only a symbolic value. Instead the new linguistics develops an interactive hermeneutic to understand the mechanisms of speech, the acquisition of language and its practical logic that constitutes the universe of meaning, because we speak as we learned to do it. The new linguistics is focused in the interests of the speaker who wants to own his own way of speaking and define the meanings in concrete situations, in contrast with a linguistics that defends the collective national interests symbolized in the shared codes.

Keywords: Linguistic Theory, Philosophy of Language, Language, Speech 


\section{El código lingüístico y el habla como praxis}

En nuestra formación como lingüistas, nos han repetido hasta el hartazgo que el lenguaje es un código, y que cada comunicación usa del código y un medio para expresar lo que tenga que decir. Eso es lo que nos dice la teoría de la información, que se necesita de la regla (= código) para la aplicación que produce el ejemplo concreto. Pero la experiencia antropológica con pueblos en aislamiento prueba lo contrario, que el código no es necesario para entenderse, y que en base a supuestos muy generales de la vida humana podemos empezar a entendernos de inmediato.

La experiencia anterior, como bagaje cultural del habla, no es necesaria, porque los acuerdos sociales válidos son los actuales y el sentido se mide siempre con los criterios actuales. Ese es el interés del hablante: definir todo y adueñarse de sus situaciones comunicativas.

[Que los criterios válidos son los actuales significa que las etimologías no tienen ningún valor comunicativo actual. Por eso las hermenéuticas que etimologizan para descubrir ciertos sentidos ocultos están descarriadas: lo que alguna vez tuvo sentido ya no lo tiene, si no adquiere validez social. Y en segundo lugar, si el sentido de una expresión o de un texto o discurso no está siendo determinado por las reglas del lenguaje, sino por experiencias personales o colectivas anteriores a los hechos, lo que antes se denominaba el contexto, entonces lo que hay es una relación causal - no de reglas - y eso normalmente se conoce como comunicación neurótica.]

La preinformación no tiene necesidad lógica. Por el contrario, plantear la necesidad del código sirve para uniformizar y por ende dominar a la gente y es de inmediato interés del estado, entendido como nación con una lengua (= código). Por eso la uniformidad del código le interesa al estado y no al hablante. Y si decimos "mi amá y mi apá" la comunicación no falla, falla la unidad de la nación y por eso se condena la expresión, por razones políticas y no por razones comunicativas. La lingüística ha creído que en los códigos había ubicado un supuesto lógico, cuando en realidad de lo que se trataba era de la sumisión social. Y eso es un hecho penoso.

\section{El código como sedimento de una práctica}

Pero lo cierto es que el código es el sedimento de una práctica, digamos así, la experiencia acumulada que nos permite decir si algo está bien dicho o no. Se nos 
dice que el código es colectivo, es de la comunidad de hablantes. El código viene a ser como el espíritu objetivo, cosificado. Pero hay una dificultad con todo eso y es que el código no está por ningún lado. No es parte de la experiencia. Si le preguntáramos a cualquier hablante cuál es su código, no sabría qué decirnos, porque no usó ninguno y tampoco podrá decirnos que el código que formulan los lingüistas es el mismo que él usa, y no podrá así validar lo que dicen los lingüistas con su propia experiencia. Simplemente porque el código de un lenguaje no es parte de la experiencia con el lenguaje. Lo que el hablante puede validar son los usos del lenguaje (nos puede dar los ejemplos de la regla pero no la regla).

\section{La lógica de la adquisición del habla}

Lo único que es parte del lenguaje es el habla, el habla que recuerda, que corrige y que explica al habla y lo que se piensa y quiere decir con el habla. Esa es la praxis del lenguaje, que se mantiene a sí misma en el mundo social que es público y que todos pueden juzgar y que, además, se transmite de una generación a otra con una lógica distinta: de una manera práctica y sin explicaciones. Adquirimos el lenguaje sin nunca usar una gramática o una teoría; por así decirlo, nos lanzamos a hablar, a imitar y a variar y probar lo que imitamos, para hacernos entender, sin nunca formular la regla explícitamente. Podemos tener el concepto, pero no expresado explícitamente, sino con ejemplos, modelos, etc. Y es importante caer en cuenta que este es otro tipo de generalización, tan válida como la abstracta con sus conceptos abstractos y reglas explícitas.

En consecuencia, las definiciones lexicales y más todavía las definiciones gramaticales no son parte del saber gramatical de los hablantes, y los hablantes no están de acuerdo acerca de las definiciones; lo que saben es qué se usa para qué y también con qué criterio distingues una cosa de otra, pero ese criterio puede ser práctico, como saber distinguir colores.

\section{La ciencia usa de la imaginación matemática para explicar el habla}

Ahora bien, la relación de las estructuras y todos los mecanismos que conforman eso que la gramática generativa llamó "la máquina de reglas" es un supuesto imaginario, que simboliza lo colectivo, como si un ejemplar de esa máquina de reglas estuviera en cada cabeza regulando la acción. Pero no lo está. La filosofía de lenguaje se encargó de probar que, aunque lo estuviera, no serviría para el propósito de explicar el habla, porque lo que tenemos en los genes, en la memoria y todo lo que digamos sigue siendo un producto individual, a menos que lo comparemos y juzguemos que es similar o distinto a otro y eso ocurre solo en 
el habla y con las reglas del habla, en público y reconocible por todos. La coincidencia requiere de un criterio para juzgar la coincidencia; y juzgar coincidencias es parte de una práctica social.

Entonces, reconocemos bien que la experiencia del lenguaje se da en el habla y que el habla está enteramente en el dominio de los hablantes, cuyas experiencias son siempre individuales, juzgables y compatibles; y que en ese hacerlas comparables, iguales y distintas, está lo colectivo, y es una práctica social. Lo social no está en que lo que se dice: "que se ha derivado de una regla colectiva", porque esa es la lógica equivocada -la lógica teórica, cuando se requería de una lógica práctica para explicar una práctica (praxis)-. Y la teoría gramatical no es parte de la experiencia individual. Eso significa que el modelo científico está mal pensado, no es así como se organiza la práctica del lenguaje.

El comportamiento regular no se explica inicialmente por la aplicación de reglas explícitas, sino de forma práctica, porque aplicar y entender reglas requiere del uso del lenguaje que se quiere explicar de ese modo. La explicación supone lo que se quiere explicar y entonces no tiene ningún valor.

¿Quiere eso decir que lo que ha dicho la lingüística no sirve? Quiere decir que la lingüística ha creado "modelos de juguete" que representan para la imaginación el comportamiento social, pero que si quiere describir la experiencia del habla o explicar cómo es que existen las regularidades del habla, se tiene que fijar en los modos prácticos con los que se logra esa regularidad. Para eso, tiene que cambiar de lógica, no solo fijarse en la formulación de reglas, que no existen en ninguna parte, y que ha sido lo que se discute entre las distintas corrientes lingüísticas.

\section{El interés por el lenguaje sedimentado}

Si nuestro interés es ocuparnos de lo colectivo, de cómo se sedimenta el lenguaje, cómo se uniformiza a los hablantes por la presión social de "hacerse entender con medios compartidos" y cómo lo social domina a lo individual, podemos hacerlo, nada lo impide.

Constataríamos, por ejemplo, que en los lenguajes naturales hay una tendencia a usar contrastes máximos para distinguir bien sonidos y nociones también, o que muchas veces se organizan grupos de elementos por un grupo de contrastes sistematizables, aunque será difícil organizar todo el lenguaje de esa manera. Y también sucede que hay reglas en competencia y ambivalencias y que a veces predominan unas u otras en distintas etapas históricas o en distintos 
contextos sociales; y se constatará muchas veces que, si hacemos un cambio en una parte, por congruencia tiende a hacerse un cambio en otra parte, aunque la conexión no sea ni directa ni evidente. Lo que quiero comunicar es que si bien las prácticas individuales son lo fundamental para la experiencia con el lenguaje, eso no quiere decir que no haya un lugar para los mecanismos sociales y que exista una dinámica entre ambas.

\section{La interacción entre la experiencia previa y el habla actual}

En casos como el encuentro con un pueblo indígena recién contactado, cuya lengua desconocemos, la práctica actual puede resultar muy limitada en sus posibilidades, si no se toma la experiencia colectiva anterior en cuenta, y eso es cierto y hay que saberlo. Pero no se piense que esa práctica actual es tan restringida, porque el habla incluye ciertos comportamientos característicos, como el comportamiento de dolor, la alegría, fruncir el ceño para concentrarse en algo, rascarse la cabeza para pensar, lamerse los labios de hambre y mucho más. Estos "comportamientos característicos" funcionan como criterios de uso en situaciones extremas, como ésta, cuando se desconoce el lenguaje y la cultura, o cuando tratamos con niños que aún no han adquirido el lenguaje oral y tenemos que fiarnos de su comportamiento para entenderlos, puesto que no podemos preguntarles lo que ocurrió o las razones, que son los criterios que maneja el lenguaje adulto. Y estos comportamientos son seguro parte del lenguaje de la especie, herencia antigua, de las razas humanas, del cromagnon y neanderthal y de los homínidos que ya tenían esas nociones en sus lenguajes naturales. Es claro que es por esos comportamientos característicos que humanos y animales nos entendemos -y mejor no te acerques a un perro que te muestra los dientes-. De manera que el lenguaje humano es producto social y especialmente del proceso de socialización que lo transmite de generación en generación; pero que, igualmente, se sustenta en la historia natural del hombre, en una cantidad innumerable de competencias que el uso del lenguaje implica, desde relacionar cosas, sacar deducciones lógicas, reconocer colores, producir tonos y reaccionar con lloros en la desgracia. De este modo, es tarea de la nueva lingüística proporcionar descripciones tan detalladas de las habilidades que requiera el habla, que éstas puedan definirse en términos fisiológicos, que den sentido a los investigadores de las funciones del cerebro. Y que se pueda así sacar a la lingüística de la etapa en que las reglas y esquemas que simbolizan las regularidades gramaticales no podían ser relacionadas con situaciones experimentales que confirmen o nieguen su existencia. 
El habla trabaja, entonces, con una díada: los usos individuales y la experiencia acumulada anterior, con toda su complejidad y hasta incongruencias internas. Pero la experiencia del pasado lista para usarse es la práctica, no es la que viene en formato de regla explícita, sino la que viene con ejemplos, con modelos, imitaciones y otras formas prácticas de inducción de una regla. Y por eso justamente es muchas veces opaca al hablante. Si un niño construye en los primeros años de su vida su propia lengua, en interacción con su entorno familiar, es él mismo el organizador de su experiencia y conoce los sentidos y usos de las expresiones que ha concertado; pero cuando cae en cuenta de que tiene que adquirir el lenguaje de uso social para entenderse y hacerse entender por un círculo mayor de personas, entonces aprende las expresiones de manera funcional, sabe para qué usarlas y qué efecto tienen, aunque desconoce su estructura interna. Es por eso que a los adultos el lenguaje les es opaco.

\section{La adquisición del lenguaje y la lógica interna del lenguaje}

Será importante, entonces, conocer cómo es que el niño adquiere el lenguaje, porque en la manera en que lo aprende está también la lógica interna del lenguaje. Así como aprendemos el lenguaje, así es. Esa lógica no tiene que ver nada con la construcción teórica, sino que es un instrumento práctico. De manera que el lenguaje se estructura en torno a ciertas habilidades donde la una supone a la otra. Esa secuencia de habilidades conforma la lógica interna del lenguaje y su universo de sentido. Esta lógica del lenguaje parte de aquello con lo que los niños y niñas vienen equipados al mundo: de sus reflejos. Entonces, la comunicación empieza con mamar, porque así reacciona el niño cuando sus labios rozán el pezón, y dejar de mamar cuando está satisfecho, a sonreír cuando reconoce ojos (personas), y chillar, cuando siente dolor o se cae, y también cuando siente ruidos, voces o luces fuertes. O cuando tienen sueño y no pueden dormir. Y los padres dirán que sus reflejos, que son automáticos, tienen otro sentido, porque ya los reconoce o porque se asusta, y es por esos malentendidos que en todas partes del mundo y en todas las culturas los padres incorporan a sus niños al universo de sentido y a la práctica social.

Eso es lo que se conoce como el proceso de socialización, el cual, si bien es consciente, no es guiado por los padres, ni tiene un currículo, ni estructura ni nada de eso; y sin embargo, es la tarea más importante, más larga y difícil que emprende un ser humano: adquirir el dominio del discurso cotidiano. Tarea que le toma cerca de 20 años y que le proporciona todos los instrumentos intelectuales para desarrollar cualquier otra tarea. 
Lo importante es que se aprende con la lógica práctica y que es un proceso abierto, multilineal y multidimensional. Tiene un motor interno que obliga al niño o niña a avanzar y avanzar vertiginosamente: hacerse entender y entender. Y esto en contextos prácticos inmediatos, para satisfacer sus necesidades básicas primero y luego las creadas por el mismo proceso: juego y afecto, y así empezar a hacerse entender y a entender a los otros y finalmente entenderse a sí mismo con esos mismos medios y técnicas que le proporciona el proceso de socialización, que avanza creando nuevas unidades de sentido, puesto que cada unidad de sentido está concertada e implica una relación social con ciertas reglas.

Es sorprendente que todas las madres digan que entiendan a sus niños y que diferencian sus lloros, pero no puedan, años más tarde, definir las unidades de sentido, salvo las más evidentes, como hambre y dolor. Esto significa que el proceso de socialización es parcialmente inconsciente y que es la manera como la especie traslada la capacidad de lenguaje y la cultura de una generación a otra. La transmisión de lengua-cultura es un recurso comparable con el de la reproducción genética y su resultado es la sociedad humana, lo que vendría a resolver la discusión acerca de la transmisión de cultura. No son ítems aislados los que propagan la cultura, es la adquisición del discurso cotidiano, con su propia lógica, que es el sustrato de toda actividad humana, y que todos los discursos usan, y que también la ciencia y la filosofía suponen, sustrato sin el cual no podrían operar.

Queda entonces claro que el lenguaje es el gran constructor de la práctica (praxis) social. El niño o niña desde un inicio tiene un desarrollo cognitivo (reconoce a personas), emocional (reacciona a luces, ruidos, se asusta por gritos), físico-sensorial (maneja el ciclo vigilia/sueño, mama, se fastidia porque requiere limpieza, coge con la mano, patalea con brazos y manos, llora por malestares y dolores y protesta cuando no lo atienden). El niño pronto requiere atención y juego y aprende a manipular a sus padres/entorno familiar para pedir más atención. Sabe entonces que tiene poder sobre otros y adquiere una relación consigo mismo. En la medida que construye nuevas unidades de sentido, va adquiriendo las funciones lógicas, como usar criterios para distinguir objetos y personas, imitar espontáneamente, reconocer regularidades y proponer cambios de reglas. Para los dos años, ya puede reconocer y proponer reglas; a los 4-5, puede jugar a extender el uso de conceptos como "animado" a objetos que sabe que no son animados y a intercambiar roles (jugar al maestro o a cambiar roles con los padres). 
Por esta etapa el niño también certifica si algunos de los supuestos del lenguaje son compartidos y entonces pregunta si la luna nos sigue o si la sombra nace de los pies. Es con este tipo de habilidades que se construye el lenguaje paso a paso. Este es un proceso en el que las unidades de sentido/relaciones sociales se ensartan como un collar, donde unas suponen a otras. Por ejemplo, no puede reprender a alguien porque no cumple lo que ha dicho, si antes no aprende a "citar" a alguien. Este tipo de lógica lo encontramos en el Libro del Tao, por el siglo VII a III antes de Cristo, como medio de explicación del desarrollo social, que allí es interpretado como sofisticación y pérdida del sentido original. En consecuencia, el lenguaje es una construcción social práctica.

\section{La visión del lenguaje}

No podemos pasar por alto el tema de la visión del lenguaje, ya que podría parecer que no me refiero al lenguaje, sino a un sistema de comunicación más simple y anterior al lenguaje, y que el lenguaje propiamente dicho empezaría recién cuando el niño aprende nombres. Pero eso es justamente lo que hay que evitar: que un prejuicio oriente nuestra percepción del lenguaje. Entre las visiones equivocadas del lenguaje, están la asunción de que las palabras representan aquello de lo que se habla, y que hoy día llamaríamos referentes (la posición del Tractatus de Wittgenstein); o la otra posición, según la cual el lenguaje está constituido por nombres, que supone que lo que se nombre ya existe o está listo antes de que venga el lenguaje a ponerle nombre, como quien pega una etiqueta a un pomo. Pero eso no siempre es cierto, pues hay experiencias en las que el lenguaje ofrece los criterios con que se distingue aquello de lo que se habla; es decir, que la experiencia es constituida por el mismo lenguaje.

Por eso es importante distinguir la lógica del lenguaje, que trata de todo aquello que es regla en el lenguaje, incluyendo los conocimientos que ayudan a distinguir "a" de "b", y que son parte de las reglas del lenguaje y que se diferencian de otros conocimientos, como qué come la especie, o cómo se reproduce, etc., aspectos que no necesariamente contribuyen a distinguir nuestra especie de otras.

Lo importante es notar que podemos hacernos distintas visiones del lenguaje -de hecho, ha sucedido ya-, y que esas visiones, existentes antes de que hagamos teoría lingüística y filosofía, son parte de la cultura y fluyen en la construcción teórica sin que seamos conscientes de ello. Estamos, pues, al borde de un nuevo riesgo: el etnocentrismo. Riesgo que invalidaría todo lo pensado. Riesgo muy real, porque por ejemplo, cuando Humboldt dice que el lenguaje 
está articulado y parte o partimos de "letras" que más o menos se equivalen con las unidades mínimas del lenguaje, los fonemas, que todavía están conectados con el sentido, nos estamos basando en la experiencia con alfabetos, y especialmente con el alfabeto griego, que es una experiencia griega y que ciertamente revolucionó y sistematizó la escritura, pero que por los estudios experimentales no tiene ninguna realidad psicológica, ni nada por el estilo: los fonemas no son parte de la experiencia de hablar que empieza más bien con la sílaba.

Es claro que los niños, cuando empiezan a gorgojear, lo primero que pronuncian son sílabas como [ta ta ta] o [ma ma ma]; eso tiene que ser así porque las consonantes solo son pronunciables en sílabas. Y no encontraremos ninguna evidencia para los morfemas como unidades mínimas; y en consecuencia, la unidad mínima del habla es la sílaba. Es la estructura de la sílaba de una lengua la que condiciona la capacidad de pronunciar una secuencia, no existe algo así como una secuencia de sonido de un morfema. Por tanto, el postulado de la articulación del lenguaje no se mantiene en su forma original con unidades mínimas, los fonemas, obviamente inspirados en el alfabeto griego. Tampoco se mantienen otros postulados de la lingüística de inicios del siglo XX, como que el cerebro solo guarda la información ordenada y sistematizada, porque si no su capacidad estaría desbordada con la cantidad de datos.

Pero el cerebro no organiza ni elabora nada por sí mismo, más bien guarda la información de manera holística, es decir, relacionada con situaciones comunicativas. No solo guarda lo que distingue un sentido del otro, una información elaborada, seleccionada, sino todos los datos, como el tono de voz y hasta la entonación, y años más tarde podemos evocar cómo un loro pronunciaba ciertas palabras. Los supuestos sobre la memoria humana estaban equivocados. Y podemos distinguir los pasos del abuelo y el ruido del motor del auto de casa. No solo los humanos. También los animales pueden realizarlo. Ese es otro prejuicio del que debemos deshacernos.

El último prejuicio del que debemos deshacernos, por ahora, es que el lenguaje es un sistema de signos. Ya hemos visto que el lenguaje no representa nada, simplemente aprendemos ciertas reglas, y regularizamos el comportamiento. Si el dolor de gases estomacales "a" me hace chillar, entonces viene ayuda "b"; puedo memorizar ese condicionamiento, y hasta repetirlo sin que el desencadenante, que son los dolores de gases, tenga lugar, todo para que me engrían, pero no es que los chillidos representen o signifiquen "dolor", ni que el niño sepa qué es dolor ni pueda distinguirlo. Eso viene después. 
El proceso de representación ideal es una invención del idealismo alemán cuyo sentido era idealizar o meter el mundo en la mente por medio de los significados. Los sentidos se definen de forma muy variada, según el tema, pero no se trata en ningún caso de algo así como una palabra y una representación unidos en un signo. En consecuencia, la lingüística adoptó de su medio social una visión cognitiva del lenguaje: que el lenguaje está para conocer y representar el mundo. Y si bien podemos asignarle al lenguaje dicha función cuando hacemos teoría, no es una función general y no sirve para explicar los usos del lenguaje.

Wittgenstein -que ensayó varias maneras para descubrir el sentido de las palabras, como ver su uso- dice en sus Investigaciones filosóficas que el sentido es lo que explica la explicación del sentido (Wittgenstein, 2001, p. 958). Tenemos que tener claro que eso puede ser varias cosas, como el sentido que quiere darle el hablante o la norma social que le da otro sentido a esa palabra. Y con esos conflictos vivimos a diario.

Caímos en cuenta del etnocentrismo de la lingüística cuando trabajamos la filosofía de lenguaje china, desde Confucio, los legistas, moístas y taoístas. Para todos ellos, el lenguaje es básicamente un conductor de relaciones sociales, sirve para manipular gente. Esta es una visión cultural del lenguaje, distinta de la eurocéntrica, que es cognitiva. Los pueblos amazónicos, que hemos conocido, por su parte, definen los sentidos más bien por lo que se llama ilustraciones gramaticales. Es decir, que un relámpago puede representar la brevedad de la vida y que un nido de abejas representa la maternidad para los harakbut. El uso de imágenes, en lugar de razones, los conecta con la comprensión emocional, distinta por cierto de la intelectual y la política. Y eso es algo que juega un rol en la práctica de la magia y la manipulación simbólica. Esta visión del lenguaje es justo lo que necesitan los curanderos.

Estas no serán las únicas visiones del lenguaje posibles. Por eso, necesitamos de un perspectivismo, porque las distintas visiones pueden haber captado aspectos válidos, pero es necesario refrendar cada visión con la experiencia que la sustente. No se trata de un relativismo total en el que todo es posible, ni es arbitrario. Hay que validar cada perspectiva. El comprobar las visiones que entran en la definición de las ciencias de las que tratamos, es, por lo tanto, un paso necesario e ineludible; si no, caemos en etnocentrismos. 
El error de la visión cognitivista occidental queda más claro cuando tomamos en cuenta que nos introducen en el uso del dolor a través del comportamiento de dolor usando al mismo niño de ejemplo. Entonces, nos dicen que nos duele y nos consuelan, hay una reacción emocional, y nos preguntan qué nos duele, qué pasó, cómo sucedió, y mucho más. Así aprendemos qué es el dolor, no como un concepto que es parte de otros fenómenos internos, o sensaciones como hambre, sed, cosquillas e irritaciones. No es el conocimiento del dolor y sus diferencias de otros conceptos similares lo que caracteriza a este conocimiento gramatical: no es teórico, parte de la reacción y del comportamiento de dolor, es una práctica. El concepto de dolor que nos da el lenguaje no tiene nada que ver con las explicaciones científicas, porque los niños no podrían entenderlas. La lógica del dolor no es el conocimiento acerca del dolor en contraste con otros fenómenos similares; la manera en que el lenguaje define el concepto es muy diferente y ese es solo el concepto ofrecido por el lenguaje. En cambio, sí tiene que ver con que si nos consuelan y distraen a veces se va el dolor. Porque hay barreras para su percepción.

Lo cierto es que la lingüística mandó los "comportamientos acompañantes", como el lenguaje de gestos, a la semiótica; no reconoció su lugar en la lógica del lenguaje, lugar que está vinculado con una primera semantización del mundo. La filosofía de lenguaje los reconoció bien, pero dejó en claro que pensar no es rascarse la cabeza, y tener hambre no es lamerse los labios; no son, pues, los criterios de uso los que definen los sentidos de las palabras. Y esto ha sido así porque la filosofía del lenguaje puso sus mayores esfuerzos en la elucidación del habla adulta, cuando la clave para comprender el rol de los "comportamietos acompañantes" estaba en la manera en que nos introducen al lenguaje, en el habla de los niños; y porque si bien reconoce estos comportamientos como una posible entrada a las reglas del lenguaje, a la "gramática profunda", no desarrolla esta posibilidad. Y lo que caracteriza a esta manera de pensar es justamente que ponemos a la adquisición de lenguaje al centro de la experiencia con el lenguaje.

\section{La revisión de la experiencia del habla y la hermenéutica del lenguaje}

Finalmente, el ejercicio que hemos hecho es la revisión de la experiencia del habla, una revisión que nos permite entender mejor la praxis del habla y en base a esa nueva comprensión plantear mejor los objetivos a lograr, cómo se interrelaciona lo que queremos estudiar con otras ciencias y cómo se complementan. 
Nuestro propósito se conectó mucho tiempo con la filosofía tardía de Ludwig Wittgenstein, pero de lo que se trata no es de buscar sustento en ninguna filosofía, cuyo propósito es resolver problemas filosóficos, sino más bien de entender los mecanismos del lenguaje cotidiano aplicándole sus propios medios, usando los mismos criterios y técnicas que se aplican en el lenguaje cotidiano.

En consecuencia, si mi pensamiento es lo que explica mi pensamiento o lo que se deduce de mi comportamiento, el sentido de mis palabras será lo que explico cuando explico el sentido o lo que se deduce de la práctica con esas palabras, las reglas establecidas. Y si sucede que lo que digo que pienso se contradice con mi forma de actuar, entonces lo que manda es mi forma de actuar. Así es la regla. Lo mismo es válido para el sentido de mis palabras, puesto que, si el sentido que les doy es distinto del que se deduce del uso establecido, el que prevalece es el uso establecido. Es así porque dar sentido o significar son casos específicos de pensar. Esto explica por qué el sentido no lo definen las definiciones, que explican lo que entendemos o queremos decir, sino que hay que ir a los usos establecidos, porque es allí donde se decide el sentido.

\section{Entender}

Se nos ha explicado que entender consiste en coincidir con el otro y en lo que se coincide es en la codificación/decodificación de los mensajes. Pero entender no consiste solo en tener coincidencias: entender es un proceso que las personas construyen desde sus supuestos, los cuales, aunque totalmente aceptados o rechazados, no dejan de ser negociados, punto que implica ciertas bases comunes que organizan el diálogo, como no contradecirse a sí mismos, por ejemplo, o que no se cambie de opinión constantemente, -con alguien así no se puede tratar, diríamos-. De manera que se puede coincidir en por qué se discrepa en algo, y se puede estar de acuerdo en estar en desacuerdo, porque se entiende dónde y por qué se opta por posiciones distintas; y que, entendido esto, se queda de la mejor manera aunque no están de acuerdo.

La lingüística tiene que definir qué es heredable y está genetizado, y eso tiene que ser una habilidad física; no se diga que se puede genetizar la gramática, hay que definir las habilidades físicas. Porque eso es lo único que puede dominar el cerebro.

A medida que entendemos mejor el lenguaje tendremos lingüísticas más específicas, más especializadas y con modelos mejor puestos a prueba. 


\section{Referencias bibliográficas}

Austin, J. L. (1962). How To Do Things With Words: Oxford University Press Chad Hansen: Philosophy of Language in Classical China: www.philosophy. hku.hk/cha/lang 4.11.2013).

Chomsky, N. (1965). Aspects of the Theory of Syntax, Cambridge, Mass.: MIT Press

Chomsky, N. (1966). Cartesian Linguistics. New York: Harper \& Row

Coseriu, E. (1962). Teoría del lenguaje y lingüística general. Madrid: Editorial Gredos.

Fodor, J. (1987). Psychosemantics: The problem of Meaning un the Philosophy of Mind. Cambridge, Mass.: MIT Press

Hegel, G.; Friedrich, W. (1971). Lógica. Traducción Antonio Zozaya. Madrid: Ricardo Aguilera Editor

Hegel, G.; Friedrich, W. (1965). System der Philosophie. Dritter Teil. Die Philosophie des Geistes. In: Sämtliche Werke. Jubiläumsasugabe in zwanzig Bänden. Zehnter Band. Stuttgart-Bad Cannstatt: Friedrich Fromann Verlag, 1965

Helberg Chávez, H. (2012). Aufgeklärte Praxis- Philosophie für das 21. Jahrhundert [La praxis lúcida - Filosofía para el siglo XXI]. CONCORDIA • Revista Internacional de Filosofía. Universidad de Aachen, 2012-62

Helberg Chávez, H. (2012). Cambio de época. Revista Solar, UNMSM

Helberg Chávez, H. (2008). Epistemología de la interculturalidad latinoamericana. Lima, UNICEF: El Vuelo de la luciérnaga, No.1

Helberg Chávez, H. (2008). Economía intercultural. La Nueva Armonía. Lima: Proyecto de Pueblos Indígenas y Desarrollo Sostenible Convenio UNMSM Universidad de Saskatchewan (Canadá).

Helberg Chávez, H. (2007). Dimensiones de la realidad. Lima: UNMSM, Facultad de Educación - Programa de Educación Intercultural Bilingüe, 445 Págs. 
Helberg Chávez, H. (2002). Fundamentación intercultural del conocimiento. Lima: Programa FORTE-PE

Helberg Chávez, H. (2002). Pedagogía de la interculturalidad. Lima: Programa FORTE-PE

Helberg Chávez, H. (1996). Mbaisik. En la penumbra del atardecer... Literatura Oral Harakmbut. Lima: CAAAP

Helberg Chávez, H. (1993). Terminología de parentesco Harakmbut. Artículo en: Amazonía Peruana, No 23, Tomo XII, pp. 107-140.

Helberg Chávez, H. (1989). Análisis funcional del verbo amarakaeri. Artículo en: Temas de Lingüística Amerindia. Primer Congreso Nacional de Investigaciones Lingüístico-Filológicas. Eds. Rodolfo Cerrón-Palomino y Gustavo Solís Fonseca. Lima: CONCYTEC-GTZ, pp. 227-249.

Helberg Chávez, H. (1984). Skizze einer Grammatik des Amarakaeri (Bosquejo de una gramática del Amarakaeri). (Tesis doctoral). Berlin. pp. 1-514

Helberg Chávez, H. (1984 a). Zur Logik von 'Geist' und 'Geistern' (Acerca del "espíritu" y los "espíritus"). Artículo en: Navicula Tubengensis. Studia in honorem Antonii Tovar. Eds. Francisco Oroz Arizcuren, Eugenio Coseriu et alium. Tübingen: Gunter Narr pp. 177-187

Kenny, A. (2008). Aristotle and Wittgenstein. Conferencia dada el 7 de noviembre 2008. Reporte por John Preston

Langacker, R. (1987). Foundations of Cognitive Grammar, vol. i. Stanford, Calif.: Stanford University Press

Lao zi. (1994). El libro del tao. Traducción, Prólogo y Notas de Juan Ignacio Reciado. Edición bilingüe. México, D.F. octava edición, 1994

Lévi-Strauss, C. (1958). Anthropologie structurale (1) . Paris: Plon, 1958

Lévi-Strauss, C. (1962). La Pensée sauvage. Paris: Plon, 1962 
Ortiz Cabanillas, P. (2002). Lenguaje y habla personal. El cerebro humano como sistema semiótico. Lima: Fondo Editorial Universidad Nacional Mayor de San Marcos

Port, R. ; Leary, A. (2005). Against Formal Phonology. Language, Valume 81, Number 4

Port, R. (2006). Words, symbols and rich memory. port@indiana.edu -http:// www.cs.indiana.edu/ port/

Port, R. (2007). How are words stored in memory? Beyond phones and phonemes, New Ideas in Psychology 25, 143.70, www.elsevier.com/locate/newideaspsych

Port, R. (2007). Phonology is not psychological and speech processing is not linguistic. Manuscrito presentado a la Society Philosophy and Psychology Meeting in Toronto, Canada, Junio 2007

Port, R. (2008). All is prosody: Phones and Phonemes are the ghosts of letters. Prosody 2008 en Campinas, Brasil, Mayo 2008

Reichel - Dolmatoff, G. (1971). Amazonian Cosmos. The Sexual and religious Symbolism of the Tukano Indians. Chicago \& London: The University of Chicago Press, 1971

Reichel - Dolmatoff, G. (1968). Desana: Simbolismo de los Indios Tukano del Vaupés. Bogotá, Universidad de los Andes y Editorial Revista Colombiana Ltda. 1968

Reichel - Dolmatoff, G. (2002). Brain and Mind in Desana Shamanism. Journal of Latin American Lore, Vol. 7, N. ${ }^{\circ}$ 1, 1981, 73-98

Reichel - Dolmatoff, G. (2003). Desana Shamans' Rock Crystals and the Hexagonal Universe. Journal of Latin American Lore, Vol. 5, N. ${ }^{\circ}$ 1, 1979, 117-128

Saffran, J. R. , Ann Senghas $\dagger$, and John C. Trueswell. (2001). The acquisition of language by children. This paper is a summary of a session presented at the 12th annual symposium on Frontiers of Science, held November 2-4, 2000, at the Arnold and Mabel Beckman Center of the National Academies of Science and Engineering in Irvine, CA. Copyright (c) 2001, The National Academy of Sciences 
Saussure, F. (1913). Cours de linguistique génerale. éd Payot (1913) 1995

Searle, J. (1969). Speech Acts. Cambridge: Cambridge University Press

Suppes, P. (1976). Syntax and Semantics of Children's Language En: W.R. Harnad, H.D. Steklis, \& J. Lancaster (Eds.), Origins and Evolution of Language and Speech, Annals of the New York Academy of Sciences, 280. New York: New York Academy of Sciences, 1976, pp. 227-237.

Wittgenstein L. (2001). Philosophische Untersuchungen. Kritisch-genetische Edition. Herausgegeben von Joachim Schulte in Zusammenarbeit mit Heikki Nyman, Eike von Savigny und Georg Henrik von Wright. Frankfurt am Main: Suhrkamp Verlag

Wittgenstein L. (1978). Bemerkungen über die Farben/ Remarks on Colour. Editadas por G.E.M. Anscombe, traducidas por Linda L. McAlister y Margarete Schättle. Edición bilingüe con un prefacio del editor. Berkeley y Los Angeles: University of California Press

Wittgenstein L. (1970). Über Gewißheit. Editado por G.E.M. Anscombe y G.H. von Wright. Frankfurt a.M.: Suhrkamp Verlag

Wittgenstein L. (1958). Schriften I. Tractatus logico-philosophicus - Tagebücher 1914-1916 - Philosophische Untersuchungen. Frankfurt a.M.: Shurkamp Verlag, 1969

Wittgenstein, L. (1988). Investigaciones filosóficas. Traducción castellana de Alfonso García Suárez y Ulises Moulines. Barcelona: Editorial Grijalbo

Winch, P. (1972). Ethics and Action. London: Routledge \& Kegan Paul 